\title{
SUBSPACE-BASED FREQUENCY ESTIMATION UTILIZING PRIOR INFORMATION
}

\author{
Petter Wirfält ${ }^{1}$, Guillaume Bouleux ${ }^{2}$, Magnus Jansson ${ }^{1}$, Petre Stoica ${ }^{3}$ \\ ${ }^{1}$ ACCESS Linnaeus Center, Electrical Engineering/Signal Processing lab \\ KTH - Royal Institute of Technology, Sweden \\ ${ }^{2}$ University of Lyon, University of Saint Etienne, LASPI, France \\ ${ }^{3}$ Systems and Control Division, Uppsala University, Uppsala, Sweden
}

\begin{abstract}
In certain frequency estimation applications one or more of the underlying frequencies are known. For example, in rotary machines the known frequency may be a strong network frequency masking important closely spaced frequencies. Being able to include this information in the design of the estimator can be expected to improve the performance when estimating such closely spaced frequencies. We present a framework to include such prior information in a class of subspace-based estimators. Through Monte Carlo simulations and real-data applications we show the usefulness of our approach.
\end{abstract}

Index Terms - Frequency estimation; Parameter estimation; Rotating machines; Failure analysis

\section{INTRODUCTION}

Frequency estimation is an important tool in a number of signal processing applications, including radar, sonar, communications, etc. Another application that the current article will look more closely at is performance monitoring of rotating mechanical systems, and especially the diagnosis of faults in the motors of such systems. In all of these situations we might have a priori known frequencies in the signals we analyze, which in the case of an electric motor might correspond to the supply frequency, known gear frequencies, etc. The crucial point is that these frequencies do not carry any information that we are interested in but might in fact degrade the estimation of the unknown, interesting, frequencies.

A number of different methods have been proposed to tackle the general sinusoids-in-noise estimation problem, but in this article we focus on eigenanalysis-based methods such as [1], [2]. Not considered in the previous references is the ability to incorporate prior knowledge of certain frequencies into the estimation of the remaining frequencies. In [3], [4] some different approaches of doing so are evaluated. Some of the approaches considered there use orthogonal projections, which we explicitly want to avoid due to the inherent information loss.

The diagnosis of rotating machines, and in particular broken rotor bars in induction motors, is a widely researched area, see, e.g. [5] for an overview of recent developments. Subspace techniques (MUSIC [6]) has previously been used in [7], [8], but DFT-methods still appear to be the prevalent diagnostic tool in the literature [5]. Recently a state-space model capturing physical parameters of the motor has been proposed in [9], where a motor failure can be detected and diagnosed from estimated model parameters.

The research leading to these results has received funding from the European Research Council under the European Community's Seventh Framework Programme (FP7/2007-2013) / ERC grant agreement \#228044
In this paper we use the Prior knowLEDGE (PLEDGE) framework of [10] in two existing frequency estimators [1], [2], [11], and show the performance gains achieved by doing so. In addition, we apply the proposed estimation method to real data for broken rotor bar detection in an asynchronous induction motor; the objective of that analysis is to estimate sideband frequencies to the network frequency in order to diagnose the machine [12].

\section{PROBLEM FORMULATION}

The frequency estimation problem can be described as follows. We observe $N$ samples of a scalar valued complex signal $y(t)$ according to

$$
y(t)=\sum_{k=1}^{d} \alpha_{k} e^{j\left(\omega_{k} t+\phi_{k}\right)}+n(t), \quad t=0, \ldots, N-1,
$$

in which, for sinusoid $k, \alpha_{k}>0$ is a real valued amplitude, $\omega_{k} \in$ $[0,2 \pi)$ is the angular frequency and $\phi_{k} \in[0,2 \pi)$ is the phase at $t=0$. Further, $\alpha_{k}$ and $\omega_{k}$ are deterministic parameters, while $\phi_{k}$ is assumed random with a uniform distribution over its possible values. The number of sinusoids, $d$, present in the signal is assumed known, and $n(t)$ is a zero mean circular Gaussian random sequence independent of $\phi_{k}$ for all $k$, with non-zero second order moment given by

$$
\mathrm{E}\left[n(t) n^{\mathrm{c}}(\tau)\right]=\sigma^{2} \delta(t, \tau)
$$

where the superscript ${ }^{\mathrm{c}}$ denotes the complex conjugate and $\delta(t, \tau)=$ 1 for $t=\tau$ and 0 otherwise. We additionally assume that a subset of $\left\{\omega_{i}\right\}_{i=1}^{d}$ is known a priori.

\section{ESTIMATOR IMPLEMENTATION}

In this section we present the general idea of the estimators later on evaluated. For more thorough explanations see [1], [11].

If we let

$$
\mathbf{x}(t)=\left[\begin{array}{lll}
\alpha_{1} e^{j\left(\omega_{1} t+\phi_{1}\right)} & \cdots & \alpha_{d} e^{j\left(\omega_{d} t+\phi_{d}\right)}
\end{array}\right]^{T},
$$

where the superscript ${ }^{T}$ denotes the transpose, we can reformulate the problem (1) into

$$
\mathbf{y}(t)=\mathbf{A x}(t)+\mathbf{n}(t), \quad t=0, \ldots, N-m
$$


in which we have defined $\mathbf{y}(t)=\left[\begin{array}{lll}y(t) & \cdots & y(t+m-1)\end{array}\right]^{T}$,

$$
\mathbf{A}=\left[\begin{array}{ccc}
1 & \cdots & 1 \\
e^{j \omega_{1}} & \cdots & e^{j \omega_{d}} \\
\vdots & & \vdots \\
e^{j(m-1) \omega_{1}} & \cdots & e^{j(m-1) \omega_{d}}
\end{array}\right]
$$

$m$ is a user parameter, and $\mathbf{n}(t)$ is defined similarly to $\mathbf{y}(t)$. Equation (4) is the standard matrix formulation for one-dimensional data, see e.g. [2]. From (3) it is clear that

$$
\mathbf{P} \triangleq \mathrm{E}\left[\mathbf{x}(t) \mathbf{x}^{*}(t)\right]=\left[\begin{array}{ccc}
\alpha_{1}^{2} & & \mathbf{0} \\
& \ddots & \\
\mathbf{0} & & \alpha_{d}^{2}
\end{array}\right],
$$

where the superscript ${ }^{*}$ denotes the conjugate transpose. Then, from (4), we find that

$$
\mathbf{R} \triangleq \mathrm{E}\left[\mathbf{y}(t) \mathbf{y}^{*}(t)\right]=\mathbf{A P A}^{*}+\sigma^{2} \mathbf{I}
$$

which is the data covariance matrix. Next, we perform an eigendecomposition of $\mathbf{R}$ and let

$$
\mathbf{E}_{s}=\left[\begin{array}{llll}
\mathbf{s}_{1} & \mathbf{s}_{2} & \cdots & \mathbf{s}_{d}
\end{array}\right]
$$

in which $\left\{\mathbf{s}_{i}\right\}_{i=1}^{d}$ correspond to the eigenvectors associated with the $d$ largest eigenvalues $\left\{\lambda_{i}\right\}_{i=1}^{d}$ of $\mathbf{R}$. With this definition of $\mathbf{E}_{s}$ we can use (7) to write

$$
\mathbf{A P A}^{*} \mathbf{E}_{s}=\mathbf{E}_{s} \tilde{\Lambda}
$$

where

$$
\tilde{\boldsymbol{\Lambda}}=\left[\begin{array}{ccc}
\lambda_{1}-\sigma^{2} & & \mathbf{0} \\
& \ddots & \\
\mathbf{0} & & \lambda_{d}-\sigma^{2}
\end{array}\right] .
$$

Now we define

$$
\boldsymbol{\beta}_{k}=\left[\begin{array}{lllllll}
\underbrace{0}_{k-1} \ldots & b_{0} & \ldots & b_{d} \underbrace{\begin{array}{llll}
0 & \ldots & 0
\end{array}}_{m-d-k}
\end{array}\right]^{T}
$$

in which $\left\{b_{i}\right\}$ are the polynomial coefficients of

$$
b_{0} z^{d}+b_{1} z^{d-1}+\ldots+b_{d}=b_{0} \prod_{i=1}^{d}\left(z-e^{j \omega_{i}}\right),
$$

the roots of which correspond to the frequencies in (1). Defining $\left\{b_{i}\right\}$ this way, it follows from (11) that $\mathbf{A}^{*} \boldsymbol{\beta}_{k}=\mathbf{0}, k=1, \ldots, m-$ $d$, and from (9), and the fact that $\mathbf{A}, \mathbf{P}$, and $\mathbf{E}_{s}$ are all full rank, that $\mathbf{E}_{s}^{*} \boldsymbol{\beta}_{k}=\mathbf{0}$. This last equality can explicitly be written for all $k$ as (see e.g. [1])

$$
\mathbf{F b} \triangleq\left[\begin{array}{ccc}
s_{1,1}^{\mathrm{c}} & \ldots & s_{1, d+1}^{\mathrm{c}} \\
\vdots & & \vdots \\
s_{d, 1}^{\mathrm{c}} & \ldots & s_{d, d+1}^{\mathrm{c}} \\
s_{1,2}^{\mathrm{c}} & \ldots & s_{1, d+2}^{\mathrm{c}} \\
\vdots & & \vdots \\
s_{1, m-d}^{\mathrm{c}} & \ldots & s_{1, m}^{\mathrm{c}} \\
\vdots & & \vdots \\
& \vdots & \\
s_{d, m-d}^{\mathrm{c}} & \ldots & s_{d, m}^{\mathrm{c}}
\end{array}\right]\left[\begin{array}{c}
b_{0} \\
\vdots \\
b_{d}
\end{array}\right]=\mathbf{0}
$$

where $s_{i, j}$ is the $j$ th element in $\mathbf{s}_{i}$, and $\mathbf{b}=\left[\begin{array}{lll}b_{0} & \cdots & b_{d}\end{array}\right]^{T}$. In practice we can only use an estimate of (7),

$$
\hat{\mathbf{R}}=\frac{1}{N-m+1} \sum_{t=0}^{N-m} \mathbf{y}(t) \mathbf{y}^{*}(t) .
$$

We then find an estimate $\hat{\mathbf{E}}_{s}$ of $\mathbf{E}_{s}$ in (8) from $\hat{\mathbf{R}}$, and hence an estimate $\hat{\mathbf{F}}$ of $\mathbf{F}$; we can then write (13) estimated from data as

$$
\hat{\mathbf{F}} \mathbf{b}=\boldsymbol{\mu},
$$

where we have accounted for the error in $\hat{\mathbf{F}}$ by introducing the residual vector $\boldsymbol{\mu}$. In [1], it is shown how to exploit the structure of $\mathbf{F}$ and $\mathbf{b}$ in (13) to arrive at an estimator of $\mathbf{b}$ which is asymptotically efficient. Having found $\mathbf{b}$, one can find the sought frequencies from (12).

The above estimator finds $\boldsymbol{\omega}$ through the polynomial coefficients b. The main idea of [10] was that a prior knowledge of some of the frequencies $\boldsymbol{\omega}$ is equivalent to knowing some factors of (12). This knowledge can easily be exploited by factoring the polynomial (12) according to

$$
b_{0} \prod_{i=1}^{d}\left(z-e^{j \omega_{i}}\right)=P_{u}(z) P_{k}(z)
$$

where $P_{k}(z)=c_{0} \prod_{i=1}^{d_{k}}\left(z-e^{j \omega_{k, i}}\right)$ is defined by the known frequencies $\left\{\omega_{k, i}\right\}_{i=1}^{d_{k}}$, and $d_{k}$ is the number of known frequencies. Reverting to vector notation, we can write

$$
\mathbf{b}=\mathbf{C} \tilde{\mathbf{b}}
$$

where $\tilde{\mathbf{b}}$ is the vector containing the polynomial coefficients of $P_{u}(z)$ corresponding to the unknown frequencies in (1), and $\mathbf{C}$ is the matrix defined by

$$
\mathbf{C}^{T}=\left[\begin{array}{cccccc}
c_{0} & c_{1} & \ldots & c_{d_{k}} & & \mathbf{0} \\
& \ddots & \ddots & & \ddots & \\
\mathbf{0} & & c_{0} & c_{1} & \ldots & c_{d_{k}}
\end{array}\right]
$$

in which the coefficients $\left\{c_{i}\right\}_{i=0}^{d_{k}}$ are the coefficients of $P_{k}(z)$. Using (17) we rewrite (15) as

$$
\hat{\mathbf{F}} \mathbf{C} \tilde{\mathbf{b}}=\boldsymbol{\mu} .
$$

In a certain sense what we have done is to filter the data $\hat{\mathbf{F}}$ by $\mathbf{C}$ in order to find a, presumably, more accurate $\tilde{\mathbf{b}}$ which only depends on the unknown frequencies. Thus we have integrated the prior frequency knowledge into the data.

Note that re-writing (15) as in (19) is not limited to the estimator developed in [1]. All estimators that use such a polynomial rooting can be expected to benefit from using the proposed PLEDGE framework: MODE [11] is such an example, where we have an expression on the form of (15) but with a different definition of $\mathbf{F}$; in [11] the data has been weighted in a specific way as to achieve asymptotical efficiency in the related direction of arrival-estimation scenario. We examine the extension of MODE as well in the following sections.

\section{SIMULATIONS}

To study the practical performance improvements offered by the method in Section 3 we perform numerical Monte Carlo simulations in which $\boldsymbol{\omega}=\left[\begin{array}{lll}2 \pi 0.5 & 2 \pi 0.52 & 2 \pi 0.56\end{array}\right]^{T}$, where we consider 


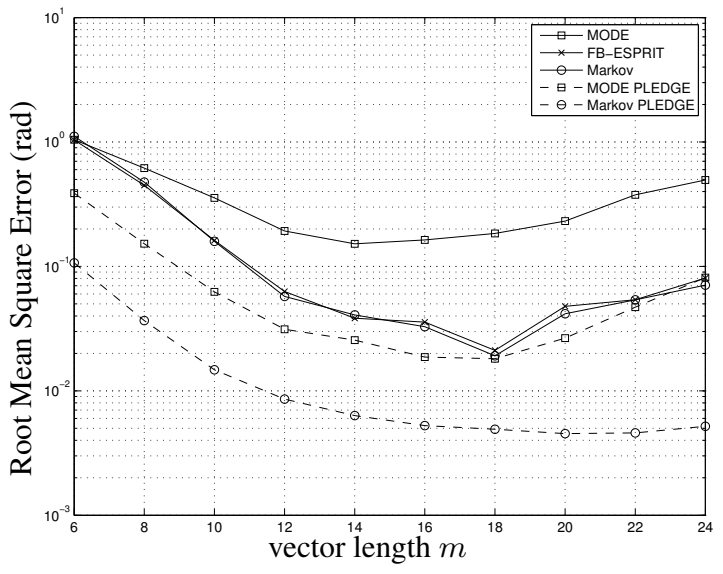

Fig. 1. RMSE of estimates corresponding to $\omega_{1}=2 \pi 0.5$, vs. $m$.

$2 \pi 0.52$ to be known. The sinusoids are equally strong with an SNR $=20 \mathrm{~dB}$, which we define as $\mathrm{SNR}_{k}=\alpha_{k}^{2} / \sigma^{2}$ for the $k$ th sinusoid. $N=30$ samples according to (1) are created, on which the respective algorithms (see below) are allowed to operate. We repeat the simulations 10000 times.

The implemented methods are: MODE as in [11]; ESPRIT (using a forward-backward averaging of data), see e.g. [1]; Markov [1], [2]; and MODE and Markov modified according to Section 3 in this paper, referred to as MODE PLEDGE and Markov PLEDGE.

In Fig. 1 we show the root mean squared error (RMSE) of the estimates corresponding to $\omega_{1}=2 \pi 0.5$ as a function of $m$, the vector length in (4). The estimates pertaining to the second unknown frequency follow the same pattern. For any value of $m$, Markov PLEDGE produces significantly more accurate results than the other methods. Since MODE is not specifically tailored for frequency estimation, it is not surprising that it gives worse results; however, MODE PLEDGE is more accurate than the methods not utilizing the prior information.

While the Esprit method is performing as well as the unmodified Markov method, it is not compatible with the PLEDGE concept as described in this paper, and we cannot inherently exploit the prior knowledge. Due to its lower computational complexity, it is otherwise an attractive estimator.

\section{BROKEN ROTOR BAR DIAGNOSIS}

We also apply the methodology described in this paper to real data acquired from the current signal extracted from an induction motor. In order to interpret the results, we give a brief background to the problem; see e.g. [13], [9] for more details.

Here we are interested in defects of rotor bars, which can be diagnosed by the slip speed of the motor. The slip speed is a measure relating the rotation speed of the motor to the current fluctuations in the stator; the stator is driving the motor rotation. The slip speed is defined by

$$
s=\frac{v_{\text {stat }}-v_{\text {rot }}}{v_{\text {stat }}},
$$

expressed in percent, where $v_{\text {rot }}$ is the actual speed and $v_{\text {stat }}$ the oscillation frequency in the stator. For industrial induction motors, the slip speed is typically between 1 and $2 \%$.

A useful indicator of broken rotor bars is based on sideband components [12], [14] around the network frequency $f_{\mathrm{NET}}$. These sidebands are located in the current spectrum at frequencies $f_{B}=$ $(1 \pm 2 k s) f_{\mathrm{NET}}$, where $k$ assumes integer values. Since $f_{\mathrm{NET}}$ is significantly stronger than all other frequencies in the spectrum, it can be expected to hamper the estimation of closely spaced frequencies; the idea then is to utilize the knowledge of $f_{\mathrm{NET}}$, in order to detect $f_{B}$ and thus to find $s$.

Comparing to, e.g., [7] the methods described herein produce frequency estimates without any associated amplitude estimate. We can however find estimates of the (complex) amplitudes based on (4), with $m=N$, according to

$$
\hat{\mathbf{x}}=\mathbf{A}_{N}^{\dagger} \mathbf{y}_{N}
$$

where the subscript $N$ denotes that we include all $N$ data-points, i.e. $\mathbf{y}_{N}=\left[\begin{array}{lll}y(0) & \cdots & y(N-1)\end{array}\right]^{T}$ and $\mathbf{A}_{N}$ defined accordingly, cf. (5). Additionally, in (21) $\mathbf{A}_{N}^{\dagger}$ denotes the Moore-Penrose pseudo-inverse of $\mathbf{A}_{N}$. The amplitude corresponding to the estimated frequency $\omega_{i}$ can thus be found as $\alpha_{i}=\left|\hat{x}_{i}\right|$.

The data analyzed consisted of $1 \cdot 10^{5}$ samples acquired with a sampling frequency of $10 \mathrm{kHz}$. For such data lengths, the DFT, with proper windowing, can be expected to resolve and correctly diagnose the side band frequencies. Thus we can use the DFT of the full data set as a benchmark to compare against.

A more interesting scenario is when fewer data points are available; hence, we use $5 \cdot 10^{3}$ data points, which we then down sample by a factor of 40 producing $N=125$ samples which we analyze with the methods proposed in Section 3. The down sampling serves two purposes: the subspace methods are more expensive to implement for large $m$, and it also narrows the frequency band within which frequency estimates are found. Choosing the largest eigenvalues of $\hat{\mathbf{R}}$ suggests $d=5$. Further, $m=62$ (roughly $N / 2$ ) is chosen.

Fig. 2 shows several plots: "Reference DFT" is the DFT spectrum based on the entire dataset, which can be seen as a reference to compare against. The proposed methods, utilizing the lesser amount of data, are also shown together with the DFT spectrum based on the reduced number of samples, "Data DFT".

We can see in Fig. 2 that the estimates produced by the proposed methods correspond to peaks in the reference spectrum. These estimated peaks match the theoretical expected values at $f=(1 \pm$ $k s) f_{\mathrm{NET}}$ for $k=1$, which are the strongest side band frequencies. Thus, both methods accurately detect the side band components, which allows an estimate of the slip speed $s$. We can also see that for the investigated data length, detection using the DFT-based method is impossible due to the inherent low resolution of that approach.

In the studied case, it seems MODE PLEDGE gives about as accurate predictions as Markov PLEDGE. Further studies are needed in order to verify the theoretical accuracy-increase of Fig. 1.

\section{CONCLUSIONS}

In this paper we have shown how to incorporate prior information into an existing class of subspace-based frequency estimators. In the simulated data cases, significant gains can be achieved through using a priori known frequency information. We also investigated the estimator performance on real data and showed that the amount of data needed to estimate relevant parameters based on frequency spectra is reduced. 


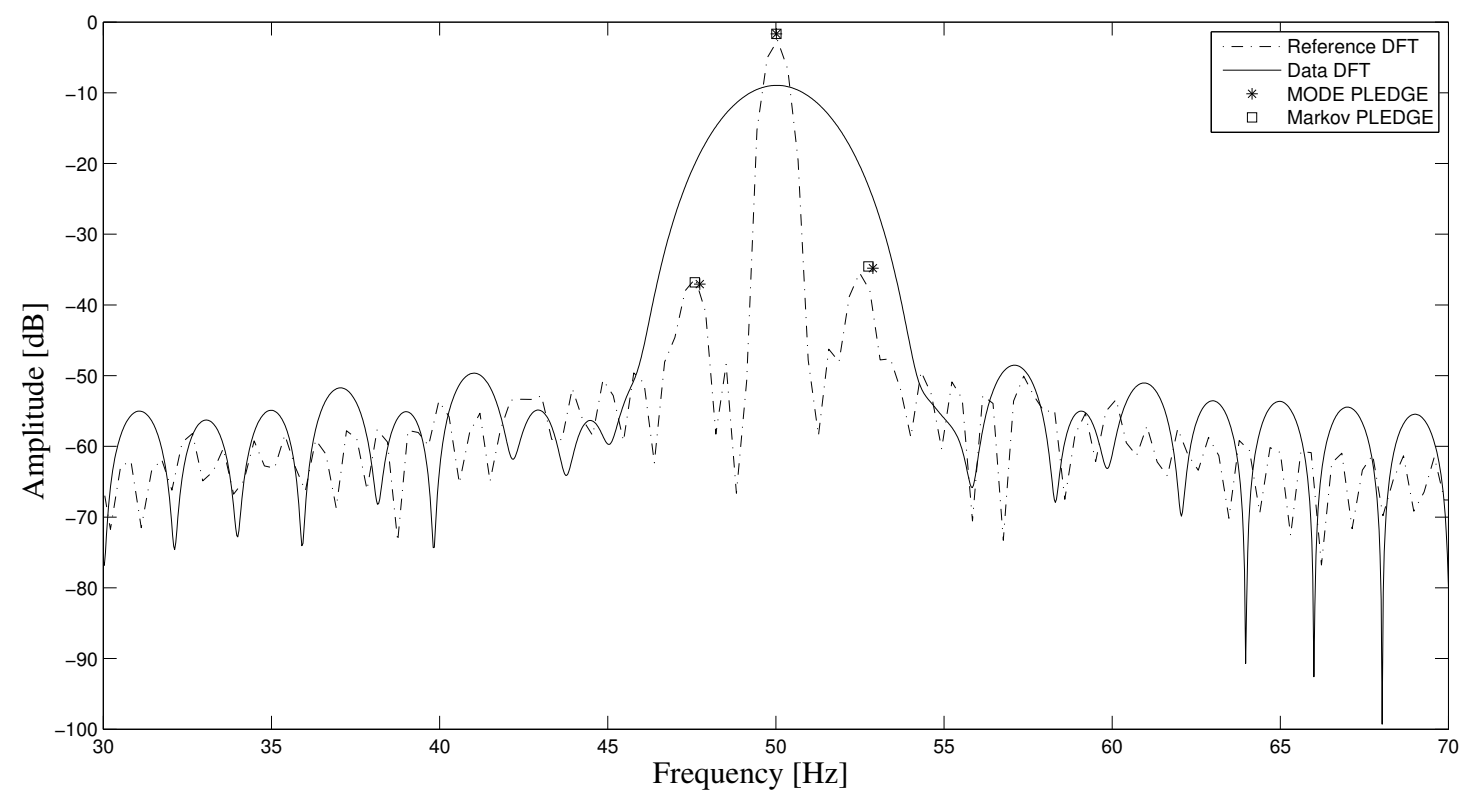

Fig. 2. Estimated frequency content of the acquired motor current signal - proposed methods and "Data DFT" are using $N=5000$ samples sampled at $10 \mathrm{kHz}$, with $m=62, d=5$. The "Reference DFT" is using the entire data set consisting of $1 \cdot 10^{5}$ samples corresponding to 10 seconds. Comparing the proposed methods to the reference, we can see that the proposed methods successfully identify the frequency peaks of interest.

\section{REFERENCES}

[1] A. Eriksson, P. Stoica, and T. Söderström, "Markov-based eigenanalysis method for frequency estimation," IEEE Trans. Signal Process., vol. 42, no. 3, pp. 586-594, Mar. 1994.

[2] M. Kristensson, M. Jansson, and B. Ottersten, "Further results and insights on subspace based sinusoidal frequency estimation,” IEEE Trans. Signal Process., vol. 49, no. 12, pp. 2962 2974, Dec. 2001.

[3] E.M. Dowling, R.D. DeGroat, and D.A. Linebarger, "Exponential parameter estimation in the presence of known components and noise," IEEE Trans. on Antennas and Propagation, vol. 42, no. 5, pp. 590-599, May 1994.

[4] D.A. Linebarger, R.D. DeGroat, E.M. Dowling, P. Stoica, and G.L. Fudge, "Incorporating a priori information into MUSICalgorithms and analysis," Signal Process., vol. 46, no. 1, pp. 85-104, 1995.

[5] A. Bellini, F. Filippetti, C. Tassoni, and G.-A. Capolino, "Advances in diagnostic techniques for induction machines," IEEE Trans. Ind. Electron., vol. 55, no. 12, pp. 4109-4126, Dec. 2008.

[6] R.O. Schmidt, "Multiple emitter location and signal parameter estimation," in Proc. of RADC Spectrum Estimation Workshop, 1979, pp. 243-258.

[7] S.H. Kia, H. Henao, and G.-A. Capolino, "A high-resolution frequency estimation method for three-phase induction machine fault detection," IEEE Trans. Ind. Electron., vol. 54, no. 4, pp. 2305-2314, Aug. 2007.
[8] F. Cupertino, E. de Vanna, L. Salvatore, and S. Stasi, "Analysis techniques for detection of IM broken rotor bars after supply disconnection," IEEE Trans. Ind. Appl., vol. 40, no. 2, pp. 526-533, Mar.-Apr. 2004.

[9] S. Bachir, S. Tnani, J.-C. Trigeassou, and G. Champenois, "Diagnosis by parameter estimation of stator and rotor faults occurring in induction machines," IEEE Trans. Ind. Electron., vol. 53, no. 3, pp. 963-973, Jun. 2006.

[10] G. Bouleux, P. Stoica, and R. Boyer, "An optimal prior knowledge-based DOA estimation method," in 17th European Signal Processing Conference, Glasgow, UK, Aug. 2009, pp. 869-873.

[11] P. Stoica and K. Sharman, "Maximum likelihood methods for direction-of-arrival estimation," IEEE Trans. Acoust., Speech, Signal Process., vol. 38, no. 7, pp. 1132-1143, Jul. 1990.

[12] J.H. Jung, J.J. Lee, and B.H. Kwon, "Online diagnosis of induction motors using MCSA," IEEE Trans. Ind. Electron., vol. 53, no. 6, pp. 1842-1852, Dec. 2006.

[13] M.E.H. Benbouzid, "A Review of Induction Motors Signature Analysis as a Medium for Faults Detection," IEEE Trans. Ind. Electron., vol. 47, no. 5, pp. 984-994, Oct. 2000.

[14] W. Deleroi, "Der Stabbruch im Käfigläufer eines Asynchronmotors," Electrical Engineering (Archiv für Elektrotechnik), vol. 67, pp. 91-99, 1984. 